Published in final edited form as:

Am J Infect Control. 2015 October 1; 43(10): 1099-1101. doi:10.1016/j.ajic.2015.05.028.

\title{
Healthcare-associated infections studies project: An American Journal of Infection Control and National Healthcare Safety Network data quality collaboration 2015 Case \#2
}

\author{
Katherine Allen-Bridson, RN, BSN, MScPH, $\mathrm{CIC}^{1}$, Angela Anttila, PhD, MSN, NPC, $\mathrm{CIC}^{2}$, \\ Janet E. Brooks, RN, BSN, CIC ${ }^{2}$, Cindy Gross, MT, SM (ASCP), CIC $^{2}$, Joan N Hebden, RN, \\ MS, $\mathrm{CIC}^{3}$, Denise Leaptrot, MSA, SM/BSMT(ASCP), $\mathrm{CIC}^{2}$, Susan Morabit, MSN, PHCNS-BC, \\ $\mathrm{CIC}^{2}$, and Marc-Oliver Wright, MT(ASCP), MS, $\mathrm{CIC}^{4}$ \\ ${ }^{1}$ National Healthcare Safety Network, Division of Healthcare Quality Promotion, Centers for \\ Disease Control and Prevention, Atlanta, GA \\ ${ }^{2} \mathrm{CACl}$, INC., Atlanta, GA \\ ${ }^{3}$ Wolters Kluwer Health-Sentri7, Bellevue, WA \\ ${ }^{4}$ Department of Infection Control, North Shore University Health System, Evanston, IL \\ ${ }^{5}$ Rollins School of Public Health of Emory University, Atlanta GA
}

This is the second case study published in a series in the American Journal of Infection Control (AJIC) since the Centers for Disease Control and Prevention/ National Healthcare

Safety Network (NHSN) surveillance definition update of 2015. These cases reflect some of the complex patient scenarios IPs have encountered in their daily surveillance of healthcareassociated infections (HAI) using NHSN definitions. Objectives have been previously published. ${ }^{(1)}$

With each case, a link to an online survey is provided, where you may answer the questions posed and receive immediate feedback in the form of answers and explanations. All individual participant answers will remain confidential, although it is the authors' intention to share a summary of the findings at a later date. Cases, answers, and explanations have been reviewed and approved by NHSN staff. We hope that you will take advantage of this offering, and we look forward to your active participation. The online survey may be found at: https://www.surveymonkey.com/s/AJIC-NHSN-2015C2

We strongly recommend that you review/reference the NHSN Patient Safety Component Manual, Device-Associated Module for information you may need to answer the case study questions. The website link is http://www.cdc.gov/nhsn/PDFs/pscManual/ 4PSC_CLABScurrent.pdf

The findings and conclusions in this case study are those of the authors and do not necessarily represent the official position of the Centers for Disease Control and Prevention.

For each question, please select the most correct answer. 


\section{Scenario 1}

Assume your facility is actively enrolled in NHSN and that your monthly reporting plan includes central line-associated bloodstream infection (CLABSI) surveillance in all ICUs, medical, surgical, and medical-surgical wards.

On January $4^{\text {th }} 2015$, a 10 -year-old female patient has been in your facility's pediatric intensive care unit (PICU) for the last 4 days. She was admitted for remission induction chemotherapy for acute myelogenous leukemia and had a peripherally inserted central catheter (PICC) inserted on admission. She weighs $25 \mathrm{~kg}$. Today, on her $3^{\text {rd }}$ day of chemotherapy, she experiences $625 \mathrm{mg}$ of diarrhea, abdominal pain and vomiting. Her absolute neutrophil count (ANC) is 400 cells $/ \mathrm{mm}^{3}$.

On January $5^{\text {th }}$, she passes a total of $500 \mathrm{mg}$ of diarrhea and vomits. Her ANC is 320 cells $/ \mathrm{mm}^{3}$.

On January $6^{\text {th }}$, her ANC is 300 cells $/ \mathrm{mm}^{3}$ and her diarrhea is improved. She has only had one episode of vomiting today.

On January $7^{\text {th }}$, she becomes disoriented and hypotensive. Her ANC level is 180 cells $/ \mathrm{mm}^{3}$. Blood is collected from her PICC line and from a peripheral venipuncture and a urine culture is also collected.

On January $8^{\text {th }}$, the blood culture, collected from the PICC line the previous day, is positive for Escherichia coli while the blood culture collected from the venipuncture site is negative. The urine culture result is negative. The patient has been afebrile since admission.

Antibiotics are started

Antibiotics continue on January $10^{\text {th }}$ and her ANC is $580 \mathrm{cells} / \mathrm{mm}^{3}$. The patient's ANC and white blood cell count (WBC) both remain above 500 cells $/ \mathrm{mm}^{3}$ for the remainder of her hospital stay.

Question 1. Which of the following statements is most correct?

a. Patient meets criterion 1 for a Laboratory Confirmed Bloodstream Infection (LCBI).

b. Patient meets criterion 1 for a Mucosal Barrier Injury-LCBI (MBI-LCBI).

c. Patient meets criterion 2 for an MBI-LCBI.

d. Patient meets criterion 2 for a Gastrointestinal Infection (GIT) and the bloodstream infection is secondary to that site of infection.

\section{Explanation}

Although on first pass the patient appears to meet criteria for a GIT infection, the symptoms used to meet the criteria, i.e., diarrhea, abdominal pain, and vomiting, are all chemotherapy side effects. The GIT infection criterion specifically states that these symptoms cannot be 
due to another, non-infectious cause ${ }^{(2)}$. Therefore they cannot be used to meet the GIT criterion.

The patient meets LCBI criterion 1 with a pathogen, not related to an infection at another site, recovered from a blood culture. The patient further meets MBI-LCBI criterion 1 since E. coli is included as an MBI-LCBI 1 organism and the patient fulfills the NHSN protocol requirements for 2 days with ANC levels below 500 cells $/ \mathrm{mm}^{3}$ in the 7-day period surrounding the blood culture (i.e., 3 days before the day of blood culture collection, the day of blood culture collection [January $7^{\text {th }}$ ] and the 3 days following blood culture collection). See Table 1, Calendar of MBI-LCBI Elements, below.

The date of the LCBI is January $7^{\text {th }}$, the date that the first element of the infection criterion occurred for the first time in the infection window period. Because the date of the LCBI was after day-2 of admission, this is a healthcare-associated infection. Since the central line was in place greater than 2 days on the date of the LCBI, this is a CLABSI LCBI-1.

The positive blood culture collected from the PICC line must be used to meet the NHSN LCBI criterion even though the blood culture collected via the venipuncture site is negative. ${ }^{(3)}$

\section{Scenario 2}

Changes from scenario 1 are bolded

On January $4^{\text {th }}$, a 10 -year-old female patient has been in your facility's PICU for the last 4 days. She was admitted for remission induction chemotherapy for acute myelogenous leukemia and had a peripherally inserted central catheter (PICC) inserted on admission. She weighs $25 \mathrm{~kg}$. Today, on her $3^{\text {rd }}$ day of chemotherapy, she experiences $625 \mathrm{mg}$ of diarrhea, abdominal pain and vomiting. Her absolute neutrophil count (ANC) is $400 \mathrm{cells} / \mathrm{mm}^{3}$.

On January $5^{\text {th }}$, she passes a total of $500 \mathrm{mg}$ of diarrhea, and has vomiting. Her ANC is 320 cells $/ \mathrm{mm}^{3}$.

On January $6^{\text {th }}$, her ANC is 300 cells $/ \mathrm{mm}^{3}$ and her diarrhea is improved. She has only had one episode of vomiting today.

On January $7^{\text {th }}$, she becomes disoriented and hypotensive. Her ANC level is 180 cells $/ \mathrm{mm}^{3}$. Blood is collected from her PICC line as well as from a peripheral venipuncture and a urine culture is also collected.

On January $8^{\text {th }}$, the blood culture, collected from the PICC line the previous day, is positive for Escherichia coli while the blood culture collected from the venipuncture site is negative. The urine culture result is negative. The patient has been afebrile since admission.

Antibiotics are started.

Antibiotics continue on January $10^{\text {th }}$ and her ANC is 580 cells $/ \mathrm{mm}^{3}$. The patient's ANC and WBC both remain above 500 cells $/ \mathrm{mm}^{3}$ for the remainder of her hospital stay. 
On January $16^{\text {th }}$, the patient becomes listless and has shaking chills. Blood cultures are collected, and the next day, Enterococcus faecium is identified in that blood culture.

NOTE: NHSN has developed a surveillance worksheet to promote consistent surveillance data collection. That worksheet, along with an example of a completed worksheet with explanation, is available under "Supporting Materials" at this site: http://www.cdc.gov/nhsn/ acute-care-hospital/clabsi/index.html Please note that there are 2 tabs at the bottom of each of these excel documents, and review information in both tabs. We recommend that you access and print these forms to use as you work through this exercise and in your routine surveillance activities.

Question 2. Which of the following statements is most correct?

a. This patient has a BSI which is not catheter-associated and which should be reported with a date of event of January $16^{\text {th }}$.

b. This patient has both a CLABSI with $E$. coli on January $7^{\text {th }}$ and a CLABSI with $E$. faecium on January $16^{\text {th }}$. Both infections are reported to NHSN.

c. The pathogen $E$. faecium should be added to the $E$. coli reported for the CLABSI dated January $7^{\text {th }}$, and not reported as a second CLABSI.

d. Patient meets criterion 2 for a Gastrointestinal Infection (GIT) and both bloodstream infections are secondary to that site of infection.

\section{Explanation}

Because the E faecium blood culture is collected during the Repeat Infection Window of the E.coli CLABSI, and E. faecium is an eligible organism for MBI-LCBI, it is not reported as a separate LCBI. Instead, the organism is added as a pathogen to the $E$. coli reported for January $7^{\text {th }}$.

\section{Scenario 3}

Changes from scenario 2 are bolded.

On January $4^{\text {th }}$, a 10 -year-old female patient has been in your facility's PICU for the last 4 days. She was admitted for remission induction chemotherapy for acute myelogenous leukemia and had a peripherally inserted central catheter (PICC) inserted on admission. She weighs $25 \mathrm{~kg}$. Today, on her $3^{\text {rd }}$ day of chemotherapy, she experiences $625 \mathrm{mg}$ of diarrhea, abdominal pain and vomiting. Her absolute neutrophil count (ANC) is 400 cells $/ \mathrm{mm}^{3}$.

On January $5^{\text {th }}$, she passes a total of $500 \mathrm{mg}$ of diarrhea, and has vomiting. Her ANC is 320 cells $/ \mathrm{mm}^{3}$.

On January $6^{\text {th }}$, her ANC is 300 cells $/ \mathrm{mm}^{3}$ and her diarrhea is improved. She has only had one episode of vomiting today. 
On January $7^{\text {th }}$, she becomes disoriented and hypotensive. Her ANC level is 180 cells $/ \mathrm{mm}^{3}$. Blood is collected from her PICC line as well as from a peripheral venipuncture and a urine culture is also collected.

On January $8^{\text {th }}$, the blood culture, collected from the PICC line the previous day, is positive for Escherichia coli while the blood culture collected from the venipuncture site is negative. The urine culture is also positive for $\mathbf{E}$. coli $>100,000 \mathrm{CFU} / \mathrm{ml}$. The patient has been afebrile since admission. Antibiotics are started.

Antibiotics continue on January $10^{\text {th }}$ and her ANC is 580 cells $/ \mathrm{mm}^{3}$. The patient's ANC and WBC both remain above 500 cells $/ \mathrm{mm}^{3}$ for the remainder of her hospital stay.

On January $16^{\text {th }}$, the patient becomes listless and has shaking chills. Blood cultures are collected, and the next day, E. faecium is identified in that blood culture.

Question 3. Which of the following statements is most correct?

a. Patient meets criteria 1 for a CLABSI with both E. coli and E. faecium.

b. Patient meets criteria for a SUTI 1a with both E. coli and E. faecium.

c. Patient meets criteria for an Asymptomatic Bacteremic Urinary Tract (ABUTI) with both E. coli and E. faecium.

d. Patient meets criterion for an ABUTI. The E. coli bloodstream infection is secondary to the ABUTI. The patient also meets criteria for a CLABSI with $E$. faecium.

\section{Explanation}

The patient, who is not catheterized, is without any of the NHSN symptoms of UTI, i.e. no fever, suprapubic tenderness, costovertebral angle pain or tenderness, urinary urgency, frequency, nor dysuria. Also at least one bacterium with a colony count of $\geq 100,000$ $\mathrm{CFU} / \mathrm{ml}$ which is cultured from the urine matches a bacterium in the blood culture. There were no more than 2 organisms with a colony count of $\geq 100,000 \mathrm{CFU} / \mathrm{ml}$ cultured from the urine which was collected on January $7^{\text {th }}$, making this an acceptable urine culture for NHSN UTI surveillance purposes. This patient meets the criteria for an ABUTI as all elements of the criterion occurred during the ABUTI infection window period. ${ }^{4}$

Only primary bloodstream infections are reported to NHSN as LCBI. The NHSN LCBI criterion states "Patient has a recognized pathogen cultured from one or more blood cultures AND organism cultured from blood is not related to an infection at another site." In this scenario, since the positive blood culture with the matching blood culture (E. coli) is an element of the ABUTI criteria, the E. coli BSI is considered secondary to the ABUTI.

During the ABUTI secondary BSI attribution period, a blood culture was collected and was positive for E. faecium. Although this blood culture was collected during the secondary BSI attribution period, E. faecium does not match the organism identified in the ABUTI, as is required to meet ABUTI criteria. ${ }^{4}$ Therefore, unless there is another site of infection to which the E. faecium BSI can be attributed as secondary, this will be a primary BSI and 
CLABSI, meeting LCBI criterion 1, for NHSN reporting purposes. This is not an MBILCBI 1 because there were not at least 2 days of ANC or WBC below 500 cells $/ \mathrm{mm}^{3}$ during the 3 days before, the day of, or 3 days after the blood culture collection date, nor is she an allogeneic hematopoietic stem cell transplant recipient within the past year.

\section{References}

1. Wright MO, Hebden JN, Bridson KA, Morrell GC, Horan T. Healthcare-associated Infections Studies Project: An American Journal of Infection Control and National Healthcare Safety Network Data Quality Collaboration. American Journal of Infection Control. 2010 Jun; 38(5):416-418. [PubMed: 20583335]

2. http://www.cdc.gov/nhsn/PDFs/pscManual/2PSC_IdentifyingHAIs_NHSNcurrent.pdf page 16

3. http://www.cdc.gov/nhsn/PDFs/pscManual/4PSC_CLABScurrent.pdf page 4-11

4. http://www.cdc.gov/nhsn/PDFs/pscManual/7pscCAUTIcurrent.pdf page 7-7 


\section{Table 1}

Calendar of MBI-LCBI Elements (applies to Scenario 1)

\begin{tabular}{|c|c|c|c|c|}
\hline & Blood Culture Collection & ANC level in cells $/ \mathrm{mm}^{3}$ & Diarrhea in mg & \\
\hline January 3 & & 525 & & \\
\hline January 4 & & 400 & 625 & \\
\hline January 5 & & 320 & 500 & $\begin{array}{l}\text { Period- Date of First } \\
\text { positive diagnostic }\end{array}$ \\
\hline January 6 & & 300 & 100 & $\begin{array}{l}\text { test } \\
\text { lOR }\end{array}$ \\
\hline January 7 & + E.coli & 180 & & First documented \\
\hline January 8 & & Not tested & & $\begin{array}{l}\text { symptom in the } \\
\text { absence of a }\end{array}$ \\
\hline January 9 & & Not tested & & $\begin{array}{l}\text { diagnostic test) plus } 3 \\
\text { days before and after }\end{array}$ \\
\hline January 10 & & 580 & & \\
\hline January 11 & & 700 & & \\
\hline
\end{tabular}

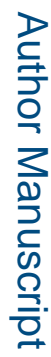

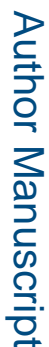

Am J Infect Control. Author manuscript; available in PMC 2016 October 01. 


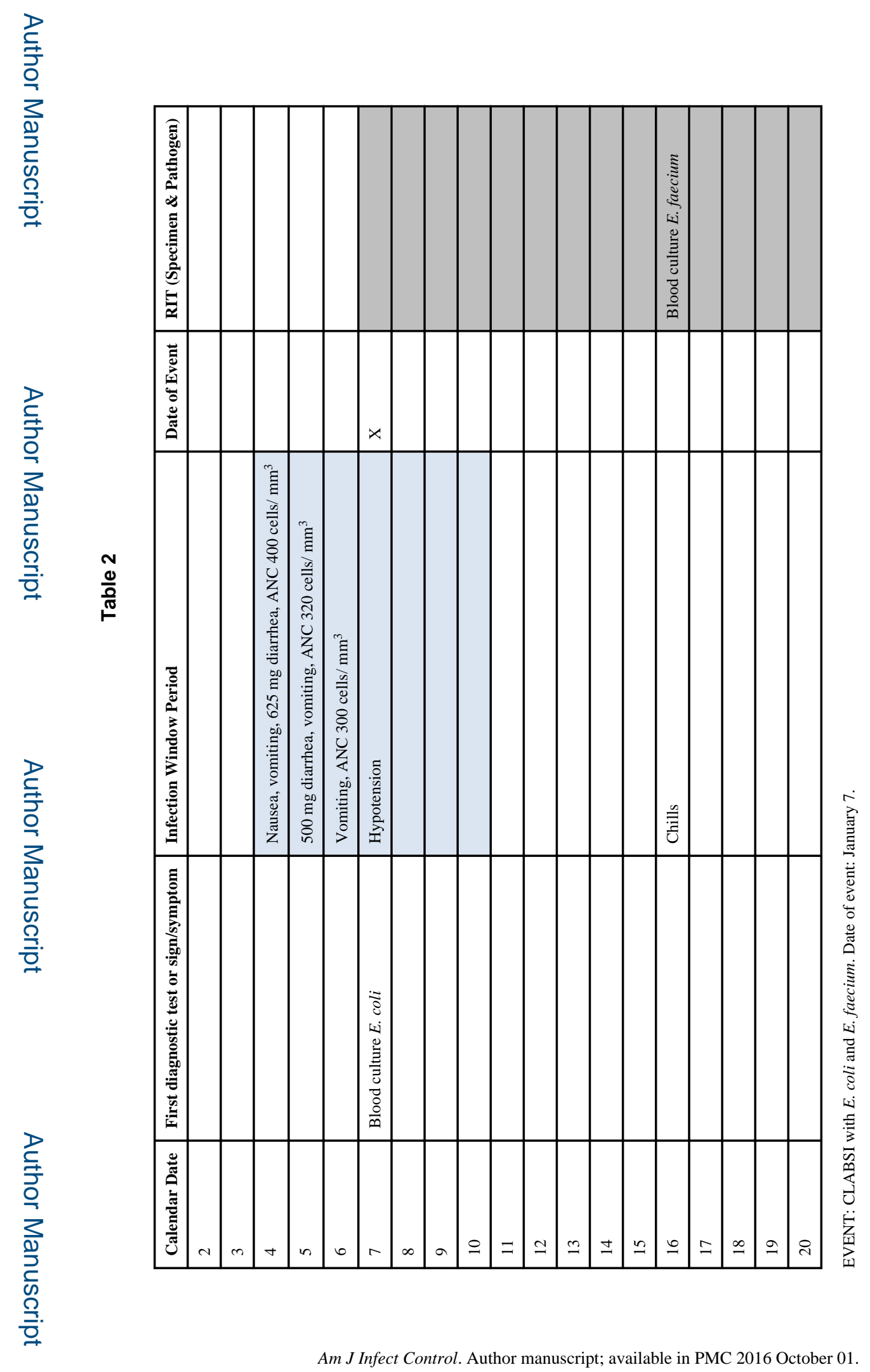




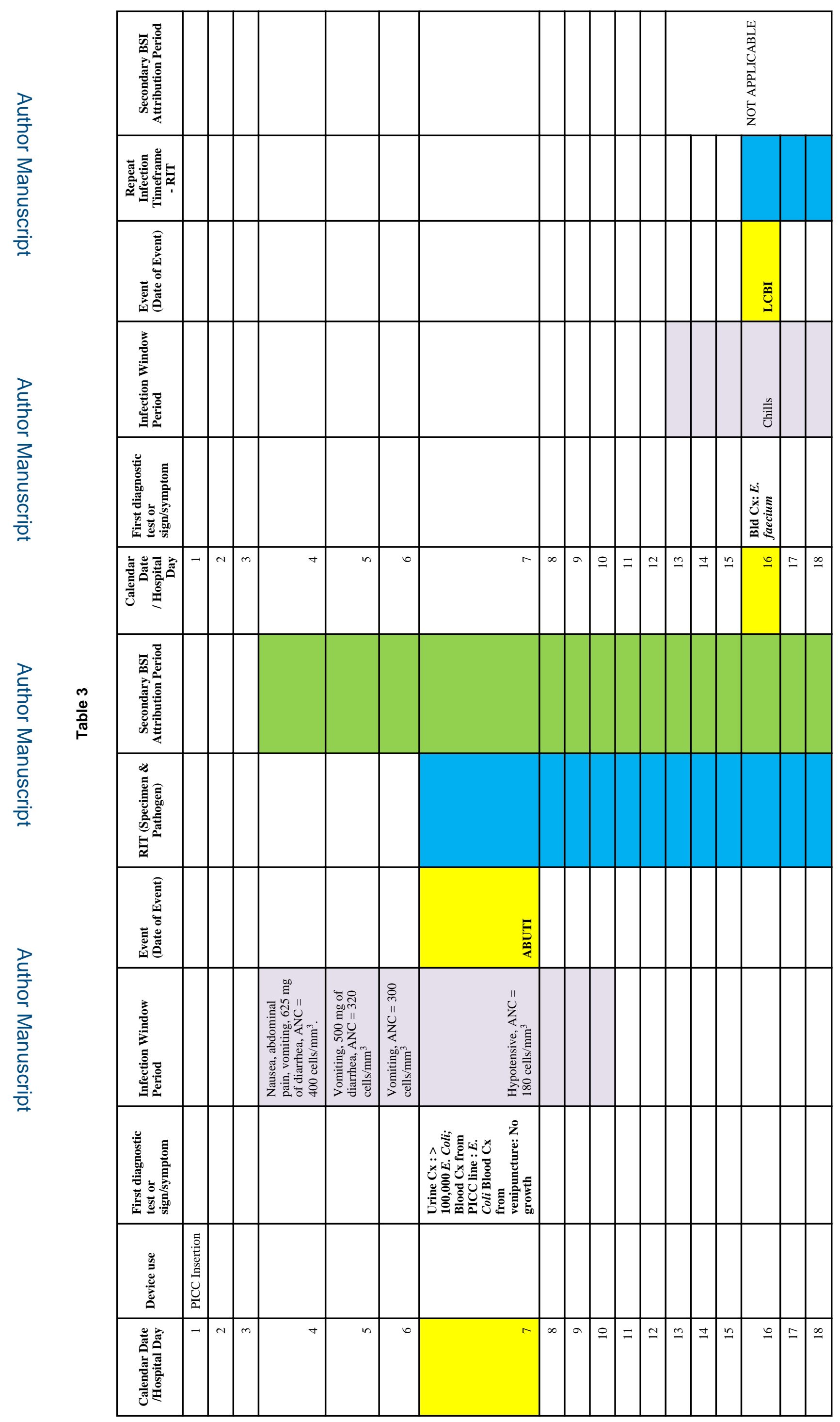


